\title{
Aprendizaje invertido con Elevator Pitch y Pecha Kucha Flipped Learning with Elevator Pitch and Pecha Kucha
}

\author{
Enrique Romero ${ }^{1}$, Jesús Sergio Artal ${ }^{2}$, José Ramón García Aranda ${ }^{3}$, Juan Manuel Artacho ${ }^{4}$ \\ \{eromero, jsartal, joser, jartacho\}@unizar.es \\ ${ }^{1}$ Dpto. Ingeniería Química y TMA \\ ${ }^{2}$ Dpto. Ingeniería Eléctrica \\ Universidad de Zaragoza \\ Zaragoza, España \\ ${ }^{3}$ Dpto. Dirección y Organización de Empresas \\ ${ }^{4}$ Dpto. Ingeniería Electrónica y Comunicaciones \\ Universidad de Zaragoza \\ Zaragoza, España
}

Resumen- La aplicación del enfoque didáctico Flipped Classroom (aula invertida) depende en gran medida de las técnicas y herramientas utilizadas en su implementación. En este trabajo se ha orientado la aplicación de la didáctica Flipped al aprovechamiento del estudio del alumno de educación superior fuera del aula con el objetivo de preparar y realizar presentaciones en clase. Se discute la utilidad de dos técnicas importantes y actuales para presentar: Elevator Pitch y Pecha Kucha. También se muestran algunas reglas y consejos sencillos en relación a la preparación de presentaciones y las ventajas e inconvenientes de aplicaciones usuales para la realización de presentaciones (PowerPoint, Prezi...). Los resultados de la aplicación de Elevator Pitch y Pecha Kucha muestran un interés evidente de los alumnos y un aprovechamiento importante del material a presentar. Se facilita una mayor y más profunda comprensión de la materia de estudio, debido a la adecuación de la exposición a las reglas impuestas, debiendo el alumno centrarse en lo más importante para conseguir una presentación más eficiente y más efectiva. La dinámica Flipped Learning se ve, por tanto, potenciada, facilitando la asimilación, reforzando el aprendizaje, y facilitando las competencias de expresión oral, síntesis, análisis, y transmisión.

\section{Palabras clave: Elevator Pitch, Pecha Kucha, Flipped, Flipped Learning, Presentación, PowerPoint, Prezi}

\begin{abstract}
The Flipped Classroom Learning strategy clearly depends on using adequate techniques and tools. This work deals with the Flipped implementation at higher education under different subjects. The out of class work has been devoted to a theme studying and the subsequent preparation of a presentation. The work at class was the presentation and later discussion. The usefulness of two good techniques for presentation (Elevator Pitch and Pecha Kucha) has been studied. Also some rules of thumb within presentation preparation and advantages and disadvantages of usual software (PowerPoint, Prezi) have been included. The use of Elevator Pitch and Pecha Kucha have showed a great student interest and good skills. It has been also observed that the students gained deeper and strong understanding of matters and proved more competences acquisition and some reason may be the necessary accommodation of materials to the two techniques rules of presentation: the student must be core-centered to make a more efficient presentation. The Flipped Learning application was then powered by the techniques and also the technologies, easing the skills in oral expression, synthesis, analysis and transmission.
\end{abstract}

Keywords: Elevator Pitch, Pecha Kucha, Flipped, Flipped Learning, Presentation, PowerPoint, Prezi

\section{INTRODUCCIÓN}

En el modelo aula invertida (Flipped Classroom), el alumnado recibe una parte instruccional de la enseñanza fuera del aula y las horas de clase se dedican a resolver dudas sobre las tareas encomendadas, discutir a fondo sobre aquello que más le cuesta comprender (aprendizaje face-to-face) o trabajar en problemas y proyectos (Bergmann, Overmyer y Wilie, 2013). Esta estrategia pedagógica busca modificar el ciclo típico de adquisición de contenidos y su aplicación, de forma que los estudiantes adquieren conocimientos necesarios antes de la clase, y los profesores guían a los estudiantes para aclarar $\mathrm{y}$ aplicar esos conocimientos en el aula de forma activa e interactiva (Figura 1). De este modo se fomenta que el alumno sea más autónomo (aprender a aprender). Los estudiantes pueden ayudarse entre sí (aprendizaje cooperativo) y el profesorado establece su rol como guía, llegando incluso a “desaparecer” en determinadas tareas autónomas. De este modo el profesor puede atender a los distintos ritmos de aprendizaje de los estudiantes. El profesor resulta más útil ya que puede corregir las tareas académicas y guiarles con sus dudas de forma inmediata, consolidando su aprendizaje en el aula (Elliott, 2014). Esta estrategia puede contribuir a mejorar el proceso de aprendizaje del estudiante, favoreciendo un aprendizaje más autónomo y significativo (O'Flaherty y Phillips, 2015). Está claro que esta nueva metodología permite reforzar el aprendizaje del estudiante y profundizar en determinados contenidos (Kerr, 2015). En cualquier caso, tanto para que el estudiante trabaje en casa como para que se pueda comprobar si este trabajo es aprovechado y se favorezca la dinámica del aula, resulta imprescindible la implementación y el uso de las herramientas TIC. Es un hecho comprobado que el estudio y la utilización de nuevos métodos interactivos atrayentes para el alumno fomenta su interés por ser partícipes de su propio aprendizaje (Porter, Graham, Spring y Welch, 2014).

La tecnología y las actividades de aprendizaje se convierten en fundamentales en este nuevo modelo al que podríamos englobar en un tipo de Blended Learning, facilitado a través de la combinación eficiente de recursos virtuales y físicos (Figura 1). Este modelo es un enfoque integral que combina la instrucción directa del alumnado con métodos constructivistas, la mejora de su comprensión conceptual o el incremento del compromiso y responsabilidad personal con su propio 
aprendizaje (Tourón, Santiago y Díez, 2014). En resumen, se está hablando de una eficacia y eficiencia pedagógica superior al sistema tradicional de clases magistrales ya que el alumnado aprende más y mejor. En la actualidad muchos autores (Bergmann y Sams, 2012; Cieliebak y Frei, 2016) han citado la relevancia que poseen los diferentes recursos y técnicas educativas en el enfoque Flipped Classroom, indicando su influencia y los beneficios obtenidos sobre el aprendizaje de los estudiantes. También han sido ampliamente debatidos los beneficios que esta nueva estrategia docente aporta frente a otras metodologías activas más “clásicas” (Bates y Galloway, 2012; Marlowe, 2012).

Un aspecto concreto muy interesante desde el punto de vista de la aplicación de la didáctica Flipped es la preparación (fuera del aula) de trabajos, individuales o en grupo, sobre un tema concreto. La realización y entrega del trabajo se complementa con la exposición ante el profesor y los demás compañeros como tareas didácticas en el aula.

Existen variadas técnicas y herramientas útiles para preparar y realizar presentaciones. La herramienta digital más utilizada en todo el mundo para este cometido es PowerPoint (o, en menor medida, otros similares). La era digital penetró en las aulas y en las empresas en gran parte gracias a esta herramienta de uso sencillo. Pero la mera utilización de PowerPoint para presentar un tema no hace que sea mejor ni que tenga mayor utilidad que una clase o presentación tradicional (la típica lección magistral en pizarra). De hecho, el abuso y el mal uso de esta aplicación puede conducir a un aprendizaje pasivo, más que a un aprendizaje más activo, como en principio se pretende (Szabo and Hastings, 2000).

También existen muchos consejos a la hora de preparar y realizar una presentación: introducir el tema, explicar la estructura de la presentación, forzar pausas, utilizar ayuda visual, hacerla corta y agradable, hacerla interactiva, lograr que la audiencia se sienta implicada y reconocida, usar la “regla de 3" (recordar siempre 3 cosas, poner 3 puntos en cada diapositiva, lanzar 3 ideas clave finales a la audiencia...), ensayarla, grabarla previamente en vídeo para detectar errores y mejorar, contar historias y anécdotas, romper el hielo en determinadas ocasiones, recordar qué diapositiva viene después ("como puede comprobarse en la siguiente diapositiva...”), tener un plan de contingencia (grabar la presentación en dos o tres dispositivos...), conocer, comprobar y preparar la sala de la presentación, confraternizar previamente con la audiencia (dejarán de ser unos “extraños”), moverse durante la presentación (el cerebro estará más oxigenado), repetir una idea clave varias veces, no hablar demasiado deprisa, hacer preguntas controvertidas y que susciten debate...

Pero además de estos consejos y trucos, existen unas técnicas a disposición del presentador, para conseguir su objetivo: captar la atención, vender un producto, una idea, etc. $\mathrm{Y}$ una buena presentación requiere de buena preparación y estudio.

Cuando alguien desea presentar algo a una audiencia, deberá estudiar bien el contexto de la actuación ("qué presentar” y “a quién presentarlo”). No es lo mismo mostrar un nuevo producto para su venta que presentar un plan de trabajo, un trabajo académico sobre un tema, ideas, competencias, habilidades, curriculum, etc. Así, el contexto determinará el procedimiento, las técnicas y las herramientas (“cómo presentarlo").

Por otro lado, el desarrollo de competencias de expresión oral en el actual contexto profesional es algo cada vez más crítico para cualquier estudiante en el ámbito de la educación superior, tanto dentro de la etapa universitaria como tal como más allá de la misma. En este ámbito, desde muchas universidades se están potenciando habilidades ligadas al emprendimiento, dado el enorme cambio que se está produciendo en el ámbito laboral a todos los niveles (Klofsten, 2000): nuevas estructuras organizativas, desaparición de los puestos de trabajo como tal para dar paso a gestores de proyectos con competencias y capacidades cada vez más cambiantes, aprendizaje a lo largo de toda la vida, etc. En ese contexto, la capacidad para transmitir ideas de forma ágil, clara y sencilla, de una manera convincente e, incluso, atrayente, se ha convertido en un elemento capital (San Tan y Frank Ng, 2006) dentro del conjunto de competencias transversales que pretenden que el estudiante sea más proactivo y emprendedor.

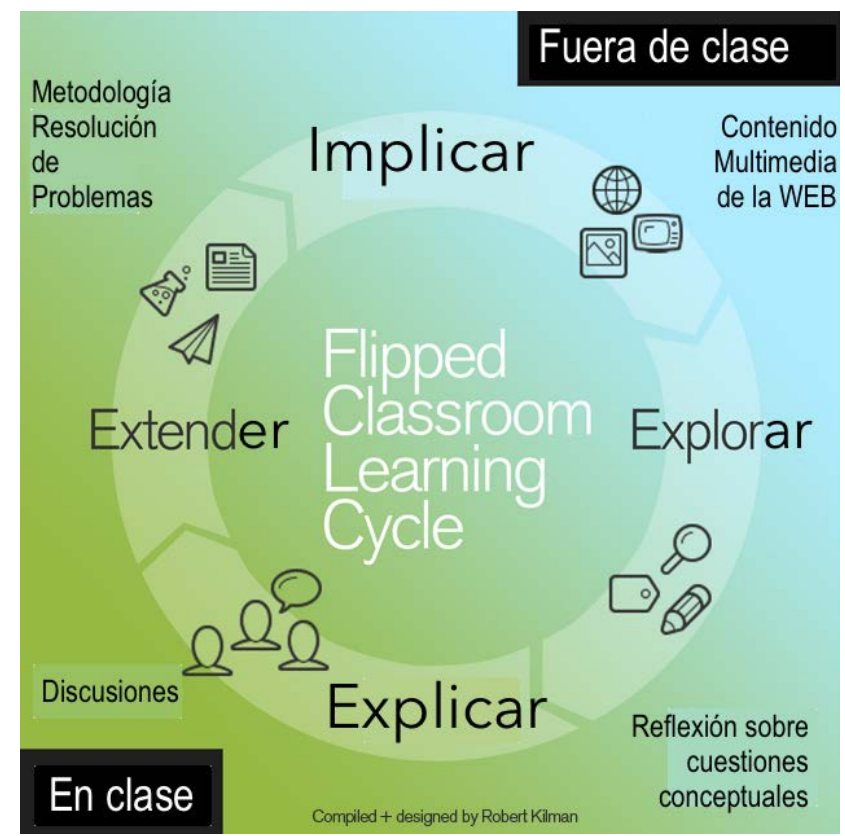

Figura 1. Ciclo de aprendizaje basado en la estrategia Flipped Classroom (Kilman, 2013).

\section{CONTEXTO}

La realización de trabajos y presentaciones es interesante para que el estudiante adquiera competencia genéricas o transversales. Se ha considerado interesante incluir varias actividades de presentación con estas técnicas con el objetivo de que los estudiantes las conozcan y las puedan emplear en su futuro desarrollo profesional. Es importante que los futuros profesionales estén al tanto de las nuevas tendencias. En ambas técnicas y ámbitos de actuación, la dinámica utilizada, desde el punto de vista del alumno, ha sido la misma:

1) estudio, reflexión, síntesis del material y preparación de una presentación como trabajo de casa

2) exposición de la presentación, con discusión, debate y profundización como trabajo de clase 
El enfoque que los alumnos debían dar a sus presentaciones estaba orientado a presentar sus trabajos no únicamente al profesor, sino también (y fundamentalmente) a sus propios compañeros. El objetivo perseguido fue el de crear debate (además de por los comentarios e indicaciones del profesor) por las aportaciones de los alumnos. La idea es, por tanto, conseguir un cierto grado de aprendizaje reflexivocooperativo, al poner en discusión entre toda la clase los trabajos individuales realizados y expuestos. Debe contener la necesidad de su realización, objetivos, contexto y público objetivo.

Durante el desarrollo de la experiencia se propusieron los siguientes objetivos educativos:

- Integrar nuevos modelos activos de enseñanzaaprendizaje basados en Flipped Classroom, introduciendo nuevos recursos y herramientas educativas junto con las tecnologías actuales en el aula.

- Analizar con detalle las mejoras incorporadas en todo el conjunto del proceso didáctico, así como las posibilidades de adaptación y transferencia a otros contextos educativos.

- Dinamizar el proceso de enseñanza-aprendizaje mediante la utilización de técnicas y herramientas educativas que promuevan un aprendizaje más activo, cooperativo, reflexivo y significativo, fomentando el trabajo no presencial.

- Motivar e incentivar al estudiante para que trabaje de forma continuada en la asignatura a lo largo del periodo académico.

- Incrementar la motivación y participación del estudiante en el aula mediante la incorporación de una nueva estrategia docente.

El enfoque didáctico Flipped surgió y creció en la educación secundaria. Sin embargo, todavía se ha aplicado muy poco en el ámbito universitario. El presente trabajo describe la experiencia vivida en distintas asignaturas de diferentes titulaciones superiores. En primer lugar, en tres asignaturas del ámbito de gestión empresarial impartidas en tres carreras diferentes durante los cursos 2015-2016 y 20162017 donde, dentro de un contexto de toma de iniciativa y fomento del espíritu emprendedor por parte de los estudiantes, se ha aplicado la técnica Elevator Pitch como instrumento de comunicación y debate de ideas, no sólo en la presentación de proyectos empresariales innovadores sino integrándola también en la dinámica natural de intervención dentro del aula. También se ha utilizado la técnica denominada Pecha Kucha en asignaturas del ámbito de la ingeniería impartidas en dos titulaciones diferentes durante el curso 2016-2017.

\section{DESCRIPCIÓN}

Este trabajo se centra en el uso de herramientas y técnicas de presentación como modo de implementación de la didáctica Flipped. El objetivo de implementar este nuevo enfoque pedagógico ha sido facilitar que las sesiones lectivas sean mucho más dinámicas, incrementando el grado de motivación de los estudiantes y permitiendo la interacción y construcción de conocimiento a través de actividades de aprendizaje activocolaborativo (Artal, Romero y Artacho, 2015). El uso de esta serie de recursos educativos, además de aumentar el factor de motivación del alumno, permite mejorar habilidades, destrezas y competencias adquiridas por los estudiantes (Perdomo, 2016).

Además de repasar algunas herramientas de presentación (PowerPoint, Prezi) se propone en este trabajo la complementación de la dinámica Flipped Learning con el desarrollo de las competencias de expresión oral, comunicación y transmisión de ideas mediante la utilización de dos de las técnicas de presentación más actuales: Elevator Pitch y Pecha Kucha. Ambas son muy utilizadas con buenos resultados desde hace unos años en ámbitos artísticos, diseño, arquitectura, económicos (como el marketing y las ventas), así como en la exposición en general de un producto, idea o proyecto (búsqueda de fondos para un determinado proyecto...).

Hay un dicho relativo a las presentaciones en general: "nadie se ha quejado nunca de que una presentación sea demasiado corta”. Efectivamente, una regla interesante para realizar presentaciones es la brevedad. Pero quizá haya que ser más precisos. O más bien... concisos. De hecho, el diccionario de la Real Academia de la Lengua Española define "concisión" como "brevedad y economía de medios en el modo de expresar un concepto con exactitud”.

La idea básica y resumida de la técnica Elevator Pitch es condensar un mensaje que llame la atención de alguien en pocos segundos o minutos, recibiendo su nombre en referencia al poco tiempo empleado, asemejando la presentación a un viaje en ascensor. Hoy se utiliza ampliamente en el mundo corporativo como herramienta estratégica, tanto para la "venta” de una idea de negocio (inicialmente solía ser un discurso de presentación sobre un proyecto o emprendimiento, ante potenciales clientes o accionistas) como para la venta de prácticamente cualquier idea en cualquier contexto (Wikipedia: “Elevator Pitch”). No existe un valor concreto sobre lo que debería durar una intervención así, si bien, el tiempo de este tipo de discursos se suele encontrar entre 30 segundos y 7 minutos.

La técnica Pecha Kucha, consiste en una presentación ajustada a un formato específico basado en la llamada "regla del 20x20”. Esto es: 20 diapositivas, 20 segundos para cada una de ellas. El tiempo disponible para una presentación es, por tanto, de 6 minutos 40 segundos. Desde el punto de vista de la educación, este formato es muy útil cuando un docente tiene muchos alumnos que exponen trabajos, El nombre proviene del "inventor” de la técnica, un japonés. Se trata de una expresión (onomatopeya) que se refiere al sonido causado por charlas cortas (en la cultura japonesa). En un principio nació en el ámbito del diseño y la arquitectura, con el objetivo de que los jóvenes estudiantes, los artistas y los arquitectos mostraran sus ideas de una forma rápida invitando a la discusión y el diálogo. Su principal ventaja: la síntesis de ideas $\mathrm{y}$ contenidos que se deben exponen, gracias al formato obligatorio. Este formato garantiza al ponente y también a su público una exposición dinámica y sistemática de todas las ideas importantes que merecen la pena ser expuestas.

La idea tuvo un éxito notable. Así que, posteriormente, el formato se extendió al mundo de los negocios. De hecho, tuvo tanto éxito que en la actualidad se celebran todos los años varios eventos denominados Pecha Kucha Nights en unas 600 ciudades de todo el mundo. Gracias a este éxito y a su difusión, la técnica también se aplica ya a casi cualquier ámbito donde cualquier comunicador tiene que presentar algo 
a una audiencia. Existen algunas variantes del Pecha Kucha. Por ejemplo, el formato 10x10: 10 diapositivas, 10 segundos en cada una de ellas. Con él la simplicidad se multiplica por dos, teniéndose que realizar todavía un mayor esfuerzo de sincretismo y sencillez en la preparación de la presentación.

En ambas técnicas, Pecha Kucha y Elevator Pitch, la presentación deber ser sencilla, pero a la vez eficaz y (en la medida de lo posible) elegante. Es decir, que capte la esencia del tema y también la atención de los espectadores. Como regla general, se debe evitar las diapositivas con mucho texto y muy cargadas (efectos, enlaces, vídeos, incrustaciones). La sencillez prima. Otro detalle importante a la hora de realizar presentaciones con estas dos técnicas es la continuidad: en general en el texto de todas las diapositivas se deberá emplear, como mucho, dos tipos de letra. Una recomendación típica es que los tipos de letra deberán ser sin rayas horizontales en los extremos de las letras: es decir, de la familia "sanserif". Ello redunda en una mayor claridad a la hora de leer el contenido en la pantalla. Las transiciones y efectos dentro de las diapositivas o entre las mismas deberá limitarse al máximo: no se distraer a la audiencia del objetivo principal (entender la esencia de la presentación). Asimismo, nunca se debe leer lo que está escrito en las diapositivas. Debe servir sólo como esquema o hilo conductor de lo que se explica. Y siempre apoya lo que el presentador quiere decir, pero nunca lo sustituye.

Existen algunos consejos desarrollados y recopilados específicamente para los que empiezan con Pecha Kucha (Clark, 2010), pero que la mayoría pueden ser aplicados también para cualquier presentación con diapositivas mediante Elevator Pitch:

- Relajarse: la audiencia sabe que sólo son 20 minutos

- Simplificar la presentación: tres puntos/ideas máximo, globalmente y por cada diapositiva

- Elegir un tema que apasione o, al menos, guste al que expone: si la presentación funciona, estupendo; si no, bueno, la audiencia notará el sentimiento y al menos lo apreciará

- $\quad$ No depender mucho de las transiciones visuales: reduce el estrés y simplifica la presentación. No obstante, el elemento visual transmite mucho: hay que tenerlo en cuenta

- $\quad$ No se deben tener notas. No hay tiempo para mirarlas.

- Disponer de un par de zonas visuales "amortiguadoras" (buffer). A tiempos de $1 / 3$ y $2 / 3$ del total de la presentación, permiten reorganizar y re-sincronizar la exposición oral con la presentación visual.

- Construir pausas en cada página. Ello previene de una exposición demasiado asfixiante para el expositor (y, probablemente, para la audiencia).

- Acudir a uno o más eventos Pecha Kucha antes de la presentación. Se podrá ver realmente en qué consiste la técnica y encontrar algunos consejos y trucos interesantes.

- Situar un "tema” visual sencillo en las diapositivas: no hay tiempo para temas complejos

- Respirar. Durante los ensayos no es tan importante, pero cuando se está en el escenario, lo es.
Las palabras clave son: sencillez, pasión y fluidez (Clark, 2010). Otros consejos aluden a la apariencia de las diapositivas:

- No intentar ajustar el discurso a lo que aparece en cada diapositiva (imaginar Pecha Kucha como una corriente de imágenes y mensajes fluyendo con la exposición oral)

- Limitarse a usar una imagen y/o un mensaje por diapositiva: no se puede rellenar la diapositiva de texto

- Utilizar un tamaño de fuente que cualquier asistente pueda leer por muy lejos que se encuentre

- $\quad$ Recurrir, siempre que se pueda, a fotografías y evitar al máximo la utilización de las imágenes prediseñadas

- Importante también es el ensayar previamente

De cara a desarrollar la capacidad de los estudiantes para transmitir de forma clara y concisa ideas (bien de negocio, bien de cualquier otra naturaleza), se seleccionaron varias materias optativas vinculadas con el concepto de emprendimiento (en carreras donde la gestión empresarial no es el elemento troncal) en diferentes facultades: “Gestión, iniciativa empresarial y marketing” (cuarto curso, Grado de Óptica y Optometría, Facultad de Ciencias, Cursos 2015-2016 (10 alumnos) y 2016-2017 (9 alumnos)); “Gestión empresarial y proyectos" (tercer curso, Grado de Física, Facultad de Ciencias, Curso 2015-2016 (15 alumnos)); “Dirección Estratégica” (primer curso, Grado de Relaciones Laborales y Recursos Humanos, Facultad de Ciencias Sociales y del Trabajo, Curso 2016-2017 (23 alumnos)). En el ámbito de las Ingenierías, se seleccionaron varias asignaturas obligatorias donde usualmente los alumnos realizan diversos trabajos sobre algún tema específico: "Procesos químicos industriales" (tercer curso, Grado de Ingeniería de Tecnologías Industriales, 43 alumnos) y “Diseño y control de convertidores” (Máster en Energías Renovables, 12 alumnos).

En el diseño de las mismas, se dio un especial peso, tanto en la evaluación como en la adquisición de competencias, al ámbito expositivo, empleando los formatos de Elevator Pitch y Pecha Kucha. Se empleó una rúbrica (basada en el Proyecto EducaLab -Ministerio de Educación, Cultura y Deporte-) atendiendo a los siguientes 5 conceptos:

- Estructura: Se reflejan, con coherencia y cohesión, las tres partes que toda intervención ha de contener: Introducción, desarrollo y final.

- Contenido: Se desarrollan todos los contenidos fundamentales conectando los puntos diferenciadores del proyecto con las "seis W", técnica vinculada a la presentación de ideas también conocida como "las cinco W y una H”: What? (¿Qué?); How? (¿Cómo?); When? (¿Cuándo?); Who? (¿Quién?); Where? (¿Dónde?); Why or for What? (¿Por qué o para qué?).

- Claridad, concisión y objetivo: Se refleja con claridad y concisión el problema que se aborda y la solución propuesta.

- Soporte: Se emplean diferentes elementos de soporte (material audiovisual, prototipos, etc.) que enriquecen notablemente la experiencia de quien escucha.

- Actitud/lenguaje no verbal: Se gestionan de manera excelente todos los aspectos clave de oratoria (pronunciación/entonación, ritmo, volumen y lenguaje no verbal/postura) 
La metodología empleada consistió en asignar proyectos/dinámicas/ejercicios de emprendimiento o que requiriesen de la defensa en público ante la clase de ideas y propuestas (en el ámbito de empresa) y asignar retos, problemas y trabajos relativos a las asignaturas (en el ámbito de la ingeniería). En cualquier caso, las etapas seguidas fueron las siguientes:

- $\quad$ Etapa de formación: Se transmite a los alumnos las bases de la herramienta y se construye, de forma colectiva, la rúbrica sobre la cual se va a evaluar el desempeño de cada uno de los estudiantes. Se abre un canal entre todos (Moodle2) donde se cuelgan y ponen a disposición de todos cualquier material complementario y/o audiovisual que permita conocer mejor la técnica y su aplicación práctica)

- Etapa de aplicación/práctica: Desde el momento en que todos conocen la herramienta, esta pasa a ser de aplicación inmediata en las diferentes dinámicas de la clase, bien en presentaciones intermedias (y finales) de los trabajos en grupo (por ejemplo, elaboración de planes de negocio innovadores relacionados con su área principal de conocimiento), exposiciones de trabajos individuales, etc.

- Etapa de retroalimentación: Tras la exposición individual, las técnicas Elevator Pitch y Pecha Kucha no terminan, sino que se extienden unos minutos más dentro de lo que sería una ronda de interpelación, opinión y/o cuestionamiento por parte del resto de estudiantes. Una vez terminada esa ronda se realiza una valoración colectiva (en base a la rúbrica diseñada) de las fortalezas y áreas de mejora del expositor, de forma que la exposición se convierte en un excelente instrumento de mejora de la habilidad (en este caso, la expresión oral eficaz).

\section{Resultados}

La mayoría de los estudiantes (63 \%) optaron por el uso de la herramienta PowerPoint, si bien el uso de Prezi va incrementándose año a año. Una de las ventajas que tiene éste frente a PowerPoint es el hecho de que mantiene en todo momento una visión de la globalidad de la presentación. Es un hecho muy valorado.

La aplicación de la rúbrica ha ayudado al profesor y a los propios alumnos en la valoración de los trabajos y presentaciones de los otros alumnos, realizándose ésta de un modo más homogéneo y ecuánime. La mejora en el desempeño de los estudiantes tras varias sesiones de práctica en la utilización de Elevator Pitch y de Pecha Kucha es sustancial, incrementándose la capacidad de oratoria de muchos de los estudiantes en la práctica totalidad de casos, dependiendo del grado de exposición “pública” que cada uno haya tenido. El cambio se percibe más cuanto antes se comienza a trabajar dicha competencia (primeros cursos), dado que muchos de los estudiantes no habían hecho exposiciones públicas antes. La rúbrica ha permitido detectar un aumento de la calidad de las presentaciones, comenzando en una mejora progresiva en la estructuración de las mismas. También se ha observado una mejora en general en cuanto a claridad y concisión, así como una mayor destreza en la definición y comprensión de los objetivos de las presentaciones. Algunos alumnos mostraron, a lo largo de la secuencia de presentaciones una mejoría notable en la expresión no verbal y actitudes durante las presentaciones, evidenciando un mayor control de sí mismos y una mayor consciencia de la utilidad de las presentaciones como muestra del trabajo de estudio previo.

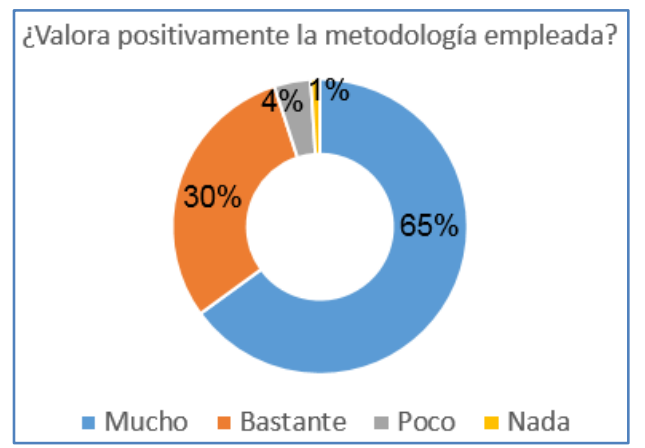

Figura 2. Resultados de la encuesta relativa a la utilización de Elevator Pitch y Pecha Kucha.

La percepción que los alumnos tienen de la utilización de las dos técnicas de presentaciones ha sido muy positiva (Figura 2). Se ha valorado en general la mejora en las competencias de análisis, síntesis y de toma de decisiones (“qué poner”, “qué no poner pero comentar”, “qué desechar”). Y, sobre todo, los estudiantes han percibido un mayor y mejor aprovechamiento de la materia de estudio mediante la necesidad previa de comprenderla en mayor profundidad para poder sintetizar y valorar lo que finalmente incluyeron en la presentación. El hecho de tener que presentar lo preparado ante el profesor, pero también ante sus propios compañeros ha supuesto un acicate para el esmero y el empeño en realizar un buen trabajo. Las aclaraciones y precisiones del profesor y también de otros compañeros han suscitado en numerosas ocasiones un debate que ha permitido profundizar en los detalles finales para un mejor entendimiento de la materia.

\section{CONCLUSIONES}

El desarrollo de esta experiencia ha permitido analizar la utilidad de Flipped Classroom complementada por otra serie de técnicas y recursos en el contexto universitario (Artal, Casanova, Serrano y Romero, 2017). Así, se han podido analizar las bondades y beneficios que ofrece esta nueva estrategia en los diversos procesos de enseñanza-aprendizaje.

Las herramientas empleadas presentan varias ventajas claves a la hora de desarrollar competencias de comunicación en los estudiantes:

- Estructuran cualquier intervención del estudiante. La inclusión natural de la técnica de "los 6W" en cualquier problemática que surja en el aula prepara a los alumnos para analizar de una forma mucho más sofisticada cualquier cuestión o tema.

- Fomentan la creatividad: Las diferentes casuísticas abordadas y las intervenciones rápidas y focalizadas permiten un ágil intercambio de opiniones e ideas que facilita que surjan otras nuevas, en un círculo virtuoso que se retroalimenta de forma natural.

- Incrementan el grado de implicación de los estudiantes ya que tienen que convencer al resto de compañeros de 
que su idea o trabajo es viable y de que es una buena idea invertir recursos financieros en ella y ponerla en marcha.

- Aumentan en los estudiantes la capacidad de concisión, brevedad y foco en lo importante que se quiere transmitir. Al extender la dinámica de Elevator Pitch y de Pecha Kucha más allá de los trabajos grupales e integrarla en la dinámica habitual del aula, prácticamente cualquier intervención se basa en hacer una defensa ágil y argumentada de la postura que se mantiene, aumentando la capacidad de pensamiento crítico, la autonomía y la proactividad.

- Aumentan la seguridad en los estudiantes, al disponer de un instrumento estructurado de diálogo constructivo, ágil y centrado en lo relevante.

- Incrementan la capacidad “dialéctica” del grupo y su capacidad de análisis y síntesis.

Por otro lado, el hecho de haber testeado la herramienta en titulaciones "lejanas" (al ámbito de la economía y la empresa) ha permitido contrastar que este tipo de instrumentos, surgidos en contextos de gestión (empresa), son perfectamente extrapolables a otros ámbitos, incluida la ingeniería, proporcionando un buen número de beneficios una vez la competencia se consolida y domina.

\section{AGRADECIMIENTOS}

Los autores agradecen a la Universidad de Zaragoza la concesión del Proyecto de Innovación Docente PIIDUZ-16090. E. Romero y J.S. Artal agradecen a la Cátedra Banco Santander-Universidad de Zaragoza la concesión del Premio Santander 2015 a la Innovación Docente.

\section{REFERENCIAS}

Artal, J.S.; Romero, E. y Artacho J.M. (2015). BlendedLearning: New trends and experiences in Higher Education. 8th International Conference of Education, Research and Innovation. ICERI15 IATED Digital Library. Seville (Spain) November 2015; pp. 7761-7771.

Artal, J.S., Casanova, O., Serrano, R.M. y Romero, E. (2017). Dispositivos móviles y Flipped Classroom. Una experiencia multidisciplinar del profesorado universitario. EDUTEC-e, Revista Electrónica de Tecnología Educativa, 59. Recuperado el 17/06/17 de http://www.edutec.es/revista

Bates, S. y Galloway, R. (2012). The inverted Classroom in a large enrolment introductory physics course: a case study. London, UK: The Higher Education Academy STEM conference. Recuperado el 30/09/16 de https://www.cs.auckland.ac.nz/courses/compsci747s2c/le ctures/paul/Bates_Galloway.pdf

Bergmann, J. \& Sams, A. (2012). Flip your Classroom: Reach every student in every class every day. Eugene, Oregon, USA: International Society for Technology in Education (ISTE).

Bergmann, J., Overmyer, J., y Wilie, B. (2013). The Flipped Class: What it is and What is Not. The Daily Riff, July 9. Recuperado el 02/10/2016 de http://bit.ly/19tQVlh

Cieliebak, M. \& Frei, A. K. (2016). Influence of Flipped Classroom on technical skills and non-technical competences of IT students. Paper presented at the Global Engineering Education Conference (EDUCON 2016). IEEExplore Digital Library. 10-13 April 2016. Abu Dhabi (United Arab Emirates) pp. 1012 - 1016.

Clark, S. (2010). Pecha Kucha tips-simplicity, flow and passion. Recuperado el 19 de junio de 2017 de https://www.buzzmaven.com/2010/03/pucha-kucha.html

Elliott, R. (2014). Do students like the Flipped Classroom? An investigation of student reaction to a Flipped undergraduate IT course. Paper presented at the Frontiers in Education Conference (FIE 2014). IEEExplore Digital Library. Recuperado el 02/10/16 de http://ieeexplore.ieee.org/document/7044070/

Kerr, B. (2015). The Flipped Classroom in engineering education: A survey of the research. International Conference on Interactive Collaborative Learning (ICL 2015). IEEExplore Digital Library. 20-24 September 2015. Florence (Italy); pp. 815 to 818.

Kilman, R. (2013). The Flipped Classroom. Teach Online. Recuperado el 19 de junio de 2017 de https://teachonline.asu.edu/2013/05/the-FlippedClassroom/

Klofsten, M. (2000). "Training entrepreneurship at universities: a Swedish case”. Journal of European Industrial Training, 24(6), 337-344.

Marlowe, C. A. (2012). The effect of the Flipped Classroom on student achievement and stress. Master of Science. Bozeman, MT: Montana State University.

O'Flaherty, J. y Phillips, C. (2015). The use of Flipped Classrooms in higher education: A scoping review. The Internet and Higher Education, 25, 85-95. Doi: 10.1016/j.iheduc.2015.02.002.

Perdomo, W. (2016). Estudio de evidencias de aprendizaje significativo en un aula bajo el modelo Flipped Classroom. EDUTEC-e, Revista Electrónica de Tecnología Educativa, 55. Recuperado el 26/09/16 de http://www.edutec.es/revista/index.php/edutece/article/view/618

Porter, W., Graham, C.R., Spring, K.A. y Welch, K.R. (2014). Blended Learning in higher education: Institutional adoption and implementation. Computers \& Education, 75, 185-195.

San Tan, S. y Frank Ng, C.K. (2006). “A problem-based Learning approach to entrepreneurship education". Education + Training, 48(6), 416-428.

Szabo, A y Hastings, N. (2000) Using IT in the undergraduate Classroom: should we replace the blackboard with PowerPoint? Computers \& Education, 35(3), 175-187

Tourón, J., Santiago, R., y Díez, A. (2014). The Flipped Classroom. Cómo convertir la escuela en un espacio de aprendizaje. Barcelona, España: Digital-text.

Wikipedia, Elevator Pitch. Recuperado el 5 de junio de 2017 de https://es.wikipedia.org/wiki/Elevator_pitch 